\title{
A kinematical search for Wolf-Rayet nebulae in the irregular galaxy IC 10
}

\author{
Almudena Bullejos ${ }^{1}$, Margarita Rosado ${ }^{1}$, Leonid N. Georgiev ${ }^{1}$, \\ César Esteban ${ }^{2}$, and J.H. Borissova ${ }^{3}$ \\ ${ }^{1}$ Instituto de Astronomía UNAM, \\ Apartado postal 70-264, 04510 México D.F., México \\ ${ }^{2}$ Instituto de Astrofísica de Canarias, La Laguna, Tenerife, España \\ ${ }^{3}$ Institute of Astronomy, Bulgarian Academy of Sciences, \\ 72 Tsarigradsko chaussèe, BG-1784 Sofia, Bulgaria
}

\begin{abstract}
In this work we report the search for nebulosities associated with the candidate Wolf-Rayet stars listed in Massey el al (1992) in the irregular galaxy IC 10, based upon $\mathrm{H} \alpha$, [S II], and [O III] direct images and imaging FabryPérot spectroscopy. Approximately $95 \%$ of the WR candidate stars we analyzed are found within nebulae. For most of these nebulae, we find broad wings on their $\mathrm{H} \alpha$ radial-velocity profiles. For some of the nebulae, the $\mathrm{H} \alpha$ velocity profiles are broadened with a typical velocity difference of $80-100 \mathrm{~km} \mathrm{~s}^{1}$.
\end{abstract}

\section{Introduction}

The irregular galaxy IC 10 has the highest surface density of Wolf-Rayet stars amongst the galaxies of the Local Group (Massey et al. 1992). IC 10 seems to be the nearest example of a starburst galaxy. IC 10 is also exceptional in having a WN/WC fraction higher than any other galaxy within the Local Group (Massey \& Armandroff 1995). If its IMF is biased towards massive stars, this could explain the high WR density, but the WN/WC ratio is completely unexpected given IC 10's metallicity (Lequeux et al. 1979) and luminosity (Klein \& Gräve 1986).

We have carried out a systematic search for optical bubble-like structures around the WR candidate stars listed in Massey et al. (1992) for the galaxy IC 10 using the PUMA Fabry-Pérot interferometer operating at the $2.1 \mathrm{~m}$ telescope at San Pedro Mártir Observatory, México. Our search for nebulae associated with the WR candidates in this galaxy is based upon $\mathrm{H} \alpha$, [S II], and [O III] direct imaging and Fabry-Pérot spectroscopy. We shall attempt to determine, through kinematical studies, whether the nebulae seen surrounding WR candidates are really associated with these stars, their precursor, or the combined effects of both the WR and precursor evolution.

\section{Results}

Our preliminary results can be sumarized as follow: 
- Approximately $95 \%$ of the WR candidates analyzed are located inside nebulae.

- In the majority of the cases, the nebulae possibly associated with WR candidates have broad radial-velocity profiles. These profiles are typically broadened with a range of velocities of $80-100 \mathrm{~km} \mathrm{~s}^{-1}$. These velocities are of the order of those found in other bubbles around WR stars. Thus, there is some evidence that these nebulae are physically associated with these WR candidates. For the rest, more work needs to be done, due to the low signal-to-noise achieved up to now.

- We also have [S II] interferometric observations of the galaxy. A kinematical study of these data is in progress.

\section{References}

Klein, U., Gräve, R. 1986, A\&AS 161, 155

Lequeux, J., Peimbert, M., Rayo, J. F., Serrano, A., Torres-Peimbert, S. 1979, A\&AS 80,155

Massey, P., Armandroff, T.E., Conti, P.S. 1992, AJ 103, 1159

Massey, P., Armandroff, T.E. 1995, AJ 109, 2470

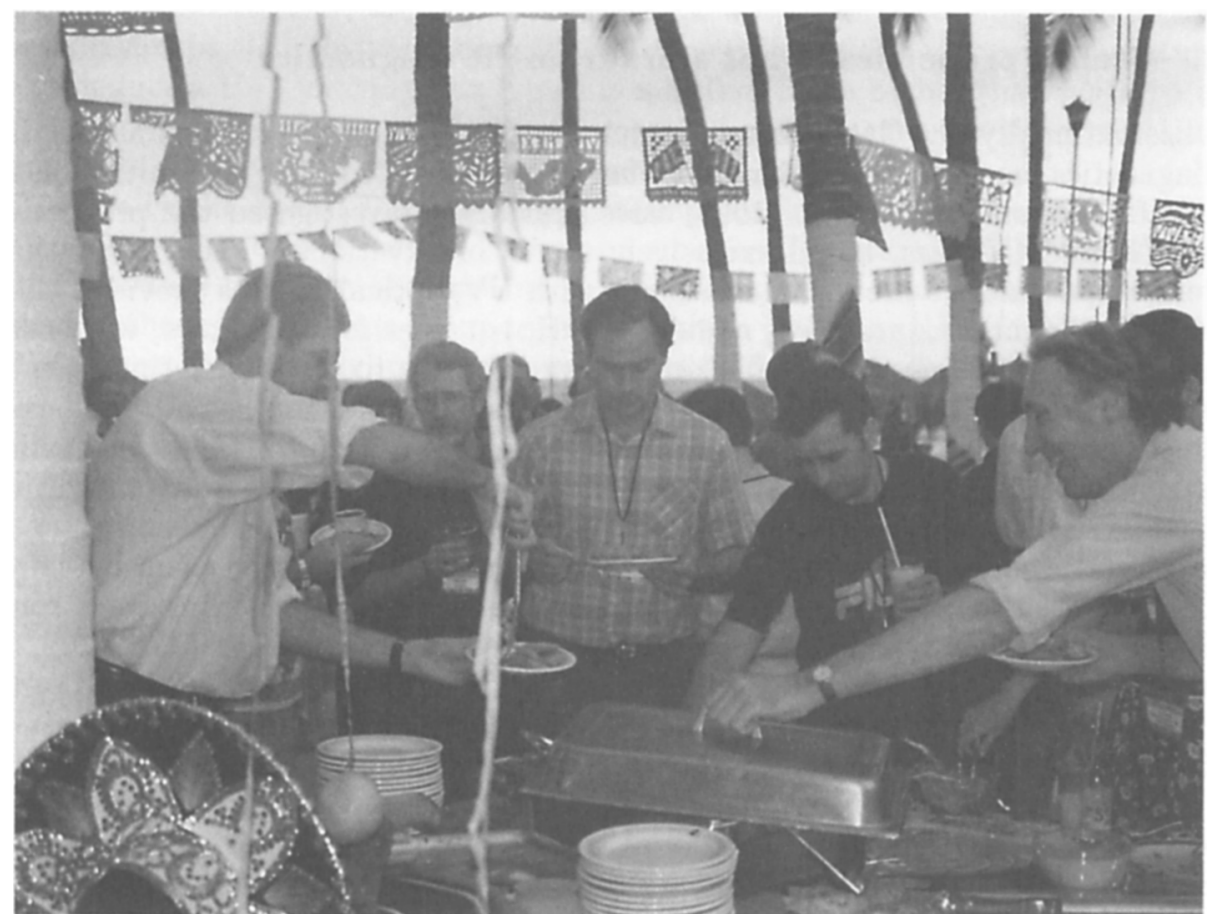

